\title{
COMMUTATORS FROM A HYPERPLANE OF MATRICES*
}

\author{
CLÉMENT DE SEGUINS PAZZIS ${ }^{\dagger}$
}

\begin{abstract}
Denote by $\mathrm{M}_{n}(\mathbb{K})$ the algebra of $n$ by $n$ matrices with entries in the field $\mathbb{K}$. A theorem of Albert and Muckenhoupt states that every trace zero matrix of $\mathrm{M}_{n}(\mathbb{K})$ can be expressed as $A B-B A$ for some pair $(A, B) \in \mathrm{M}_{n}(\mathbb{K})^{2}$. Assuming that $n>2$ and that $\mathbb{K}$ has more than 3 elements, it is proved that the matrices $A$ and $B$ can be required to belong to an arbitrary given hyperplane of $\mathrm{M}_{n}(\mathbb{K})$.
\end{abstract}

Key words. Commutator, Trace, Hyperplane, Matrices.

AMS subject classifications. 15A24, 15A30.

\section{Introduction.}

1.1. The problem. In this article, we let $\mathbb{K}$ be an arbitrary field. We denote by $\mathrm{M}_{n}(\mathbb{K})$ the algebra of square matrices with $n$ rows and entries in $\mathbb{K}$, and by $\mathfrak{s l}_{n}(\mathbb{K})$ its hyperplane of trace zero matrices. The trace of a matrix $M \in \mathrm{M}_{n}(\mathbb{K})$ is denoted by $\operatorname{tr} M$. Given two matrices $A$ and $B$ of $\mathrm{M}_{n}(\mathbb{K})$, one sets

$$
[A, B]:=A B-B A,
$$

known as the commutator, or Lie bracket, of $A$ and $B$. Obviously, $[A, B]$ belongs to $\mathfrak{s l}_{n}(\mathbb{K})$. Although it is easy to see that the linear subspace spanned by the commutators is $\mathfrak{s l}_{n}(\mathbb{K})$, it is more difficult to prove that every trace zero matrix is actually a commutator, a theorem which was first proved by Shoda 9 for fields of characteristic 0, and later generalized to all fields by Albert and Muckenhoupt 11. Recently, exciting new developments on this topic have appeared; most notably, the long-standing conjecture that the result holds for all principal ideal domains has just been solved by Stasinski [10] (the case of integers had been worked out earlier by Laffey and Reams [5]).

Here, we shall consider the following variation of the above problem:

Given a (linear) hyperplane $\mathcal{H}$ of $\mathrm{M}_{n}(\mathbb{K})$, is it true that every trace zero matrix is the commutator of two matrices of $\mathcal{H}$ ?

\footnotetext{
* Received by the editors on July 7, 2013. Accepted for publication on February 2, 2014. Handling Editor: Raphael Loewy.

†Université de Versailles Saint-Quentin-en-Yvelines, Laboratoire de Mathématiques de Versailles, 45 avenue des Etats-Unis, 78035 Versailles cedex, France (dsp.prof@gmail.com).
} 
Our first motivation is that this constitutes a natural generalization of the following result of Thompson:

Theorem 1.1 (Thompson, Theorem 5 of [1]). Assume that $n \geq 3$. Then, $\left[\mathfrak{s l}_{n}(\mathbb{K}), \mathfrak{s l}_{n}(\mathbb{K})\right]=\mathfrak{s l}_{n}(\mathbb{K})$.

Another motivation stems from the following known theorem:

TheOREM 1.2 (Proposition 4 of [8]). Let $\mathcal{V}$ be a linear subspace of $\mathrm{M}_{n}(\mathbb{K})$ with $\operatorname{codim} \mathcal{V}<n-1$. Then, $\mathfrak{s l}_{n}(\mathbb{K})=\operatorname{span}\left\{[A, B] \mid(A, B) \in \mathcal{V}^{2}\right\}$.

Thus, a natural question to ask is whether, in the above situation, every trace zero matrix is a commutator of two matrices of $\mathcal{V}$. Studying the case of hyperplanes is an obvious first step in that direction (and a rather non-trivial one, as we shall see).

An additional motivation is the corresponding result for products (instead of commutators) that we have obtained in [8]:

TheOREM 1.3 (Theorem 3 of 8$]$ ). Let $\mathcal{H}$ be a (linear) hyperplane of $\mathrm{M}_{n}(\mathbb{K})$, with $n>2$. Then, every matrix of $\mathrm{M}_{n}(\mathbb{K})$ splits up as $A B$ for some $(A, B) \in \mathcal{H}^{2}$.

1.2. Main result. In the present paper, we shall prove the following theorem:

THEOREM 1.4. Assume that $\# \mathbb{K}>3$ and $n>2$. Let $\mathcal{H}$ be an arbitrary hyperplane of $\mathrm{M}_{n}(\mathbb{K})$. Then, every trace zero matrix of $\mathrm{M}_{n}(\mathbb{K})$ splits up as $A B-B A$ for some $(A, B) \in \mathcal{H}^{2}$.

Let us immediately discard an easy case. Assume that $\mathcal{H}$ does not contain the identity matrix $I_{n}$. Then, given $(A, B) \in \mathrm{M}_{n}(\mathbb{K})^{2}$, we have

$$
\left[\lambda I_{n}+A, \mu I_{n}+B\right]=[A, B]
$$

for all $(\lambda, \mu) \in \mathbb{K}^{2}$, and obviously there is a unique pair $(\lambda, \mu) \in \mathbb{K}^{2}$ such that $\lambda I_{n}+A$ and $\mu I_{n}+B$ belong to $\mathcal{H}$. In that case, it follows from the Albert-Muckenhoupt theorem that every matrix of $\mathfrak{s l}_{n}(\mathbb{K})$ is a commutator of matrices of $\mathcal{H}$. Thus, the only case left to consider is the one when $I_{n} \in \mathcal{H}$. As we shall see, this is a highly nontrivial problem. Our proof will broadly consist in refining Albert and Muckenhoupt's method.

The case $n=2$ can be easily described over any field:

Proposition 1.5. Let $\mathcal{H}$ be a hyperplane of $\mathrm{M}_{2}(\mathbb{K})$.

(a) If $\mathcal{H}$ contains $I_{2}$, then $[\mathcal{H}, \mathcal{H}]$ is a 1 -dimensional linear subspace of $\mathrm{M}_{2}(\mathbb{K})$.

(b) If $\mathcal{H}$ does not contain $I_{2}$, then $[\mathcal{H}, \mathcal{H}]=\mathfrak{s l}_{2}(\mathbb{K})$.

Proof. Point (b) has just been explained. Assume now that $I_{2} \in \mathcal{H}$. Then, there 
are matrices $A$ and $B$ such that $\left(I_{2}, A, B\right)$ is a basis of $\mathcal{H}$. For all $\left(a, b, c, a^{\prime}, b^{\prime}, c^{\prime}\right) \in \mathbb{K}^{6}$, one finds

$$
\left[a I_{2}+b A+c B, a^{\prime} I_{2}+b^{\prime} A+c^{\prime} B\right]=\left(b c^{\prime}-b^{\prime} c\right)[A, B] .
$$

Moreover, as $A$ is a $2 \times 2$ matrix and not a scalar multiple of the identity, it is similar to a companion matrix, whence the space of all matrices which commute with $A$ is $\operatorname{span}\left(I_{2}, A\right)$. This yields $[A, B] \neq 0$. As obviously $\mathbb{K}=\left\{b c^{\prime}-b^{\prime} c \mid\left(b, c, b^{\prime}, c^{\prime}\right) \in \mathbb{K}^{4}\right\}$, we deduce that $[\mathcal{H}, \mathcal{H}]=\mathbb{K}[A, B]$ with $[A, B] \neq 0$.

\subsection{Additional definitions and notation.}

- Given a subset $\mathcal{X}$ of $\mathrm{M}_{n}(\mathbb{K})$, we set

$$
[\mathcal{X}, \mathcal{X}]:=\left\{[A, B] \mid(A, B) \in \mathcal{X}^{2}\right\} .
$$

- The canonical basis of $\mathbb{K}^{n}$ is denoted by $\left(e_{1}, \ldots, e_{n}\right)$.

- Given a basis $\mathcal{B}$ of $\mathbb{K}^{n}$, the matrix of coordinates of $\mathcal{B}$ in the canonical basis of $\mathbb{K}^{n}$ is denoted by $P_{\mathcal{B}}$.

- Given $i$ and $j$ in $\llbracket 1, n \rrbracket$, one denotes by $E_{i, j}$ the matrix of $\mathrm{M}_{n}(\mathbb{K})$ with all entries zero except the one at the $(i, j)$-spot, which equals 1.

- A matrix of $\mathrm{M}_{n}(\mathbb{K})$ is cyclic when its minimal polynomial has degree $n$ or, equivalently, when it is similar to a companion matrix.

- The $n$ by $n$ nilpotent Jordan matrix is denoted by

$$
J_{n}=\left[\begin{array}{cccc}
0 & 1 & & (0) \\
& \ddots & \ddots & \\
& & \ddots & 1 \\
(0) & & & 0
\end{array}\right]
$$

- A Hessenberg matrix is a square matrix $A=\left(a_{i, j}\right) \in \mathrm{M}_{n}(\mathbb{K})$ in which $a_{i, j}=0$ whenever $i>j+1$. In that case, we set

$$
\ell(A):=\left\{j \in \llbracket 1, n-1 \rrbracket: a_{j+1, j} \neq 0\right\} .
$$

- One equips $\mathrm{M}_{n}(\mathbb{K})$ with the non-degenerate symmetric bilinear form

$$
b:(M, N) \mapsto \operatorname{tr}(M N),
$$

to which orthogonality refers in the rest of the article.

Given $A \in \mathrm{M}_{n}(\mathbb{K})$, one sets

$$
\operatorname{ad}_{A}: M \in \mathrm{M}_{n}(\mathbb{K}) \mapsto[A, M] \in \mathrm{M}_{n}(\mathbb{K}),
$$


which is an endomorphism of the vector space $\mathrm{M}_{n}(\mathbb{K})$; its kernel is the centralizer

$$
\mathcal{C}(A):=\left\{M \in \mathrm{M}_{n}(\mathbb{K}): A M=M A\right\}
$$

of the matrix $A$. Recall the following nice description of the range of $\operatorname{ad}_{A}$, which follows from the rank theorem and the basic observation that $\operatorname{ad}_{A}$ is skew-symmetric for the bilinear form $(M, N) \mapsto \operatorname{tr}(M N)$ :

Lemma 1.6. Let $A \in \mathrm{M}_{n}(\mathbb{K})$. The range of $\operatorname{ad}_{A}$ is the orthogonal of $\mathcal{C}(A)$, that is the set of all $N \in \mathrm{M}_{n}(\mathbb{K})$ for which

$$
\forall B \in \mathcal{C}(A), \operatorname{tr}(B N)=0 .
$$

In particular, if $A$ is cyclic then its centralizer is $\mathbb{K}[A]=\operatorname{span}\left(I_{n}, A, \ldots, A^{n-1}\right)$, whence $\operatorname{im}\left(\operatorname{ad}_{A}\right)$ is defined by a set of $n$ linear equations:

LEMma 1.7. Let $A \in \mathrm{M}_{n}(\mathbb{K})$ be a cyclic matrix. The range of $\operatorname{ad}_{A}$ is the set of all $N \in \mathrm{M}_{n}(\mathbb{K})$ for which

$$
\forall k \in \llbracket 0, n-1 \rrbracket, \operatorname{tr}\left(A^{k} N\right)=0 .
$$

REMARK 1. Interestingly, the two special cases below yield the strategy for Shoda's approach and Albert and Muckenhoupt's, respectively:

(i) Let $D$ be a diagonal matrix of $\mathrm{M}_{n}(\mathbb{K})$ with distinct diagonal entries. Then, the centralizer of $D$ is the space $\mathcal{D}_{n}(\mathbb{K})$ of all diagonal matrices, and hence, $\operatorname{imad}_{D}$ is the space of all matrices with diagonal zero. As every trace zero matrix that is not a scalar multiple of the identity is similar to a matrix with diagonal zero 4, Shoda's theorem of [9] follows easily.

(ii) Consider the case of the Jordan matrix $J_{n}$. As $J_{n}$ is cyclic, Lemma 1.7yields that $\operatorname{im}\left(\operatorname{ad}_{J_{n}}\right)$ is the set of all matrices $A=\left(a_{i, j}\right) \in \mathrm{M}_{n}(\mathbb{K})$ for which $\sum_{k=1}^{n-\ell} a_{k+\ell, k}=0$ for all $\ell \in \llbracket 0, n-1 \rrbracket$. In particular, if $A=\left(a_{i, j}\right) \in \mathrm{M}_{n}(\mathbb{K})$ is Hessenberg, then this condition is satisfied whenever $\ell>1$, and hence, $A \in \operatorname{im}\left(\operatorname{ad}_{J_{n}}\right)$ if and only if $\operatorname{tr} A=0$ and $\sum_{k=1}^{n-1} a_{k+1, k}=0$. Albert and Muckenhoupt's proof is based upon the fact that, except for a few special cases, the similarity class of a matrix must contain a Hessenberg matrix $A$ that satisfies the extra equation $\sum_{k=1}^{n-1} a_{k+1, k}=0$.

\section{Proof of the main theorem.}

2.1. Proof strategy. Let $\mathcal{H}$ be a hyperplane of $\mathrm{M}_{n}(\mathbb{K})$. We already know that $[\mathcal{H}, \mathcal{H}]=\mathfrak{s l}_{n}(\mathbb{K})$ if $I_{n} \notin \mathcal{H}$. Thus, in the rest of the article, we will only consider the case when $I_{n} \in \mathcal{H}$. 
Our proof will use three basic but potent principles:

(1) Given $A \in \mathfrak{s l}_{n}(\mathbb{K})$, if some $A_{1} \in \mathcal{H}$ satisfies $A \in \operatorname{im}\left(\operatorname{ad}_{A_{1}}\right)$ and $\mathcal{C}\left(A_{1}\right) \not \subset \mathcal{H}$, then $A \in[\mathcal{H}, \mathcal{H}]$. Indeed, in that situation, we find $A_{2} \in \mathrm{M}_{n}(\mathbb{K})$ such that $A=\left[A_{1}, A_{2}\right]$, together with some $A_{3} \in \mathcal{C}\left(A_{1}\right)$ for which $A_{3} \notin \mathcal{H}$. Then, the affine line $A_{2}+\mathbb{K} A_{3}$ is included in the inverse image of $\{A\}$ by $\operatorname{ad}_{A_{1}}$ and it has exactly one common point with $\mathcal{H}$.

(2) Let $(A, B) \in \mathfrak{s l}_{n}(\mathbb{K})^{2}$ and $\lambda \in \mathbb{K}$. If there are matrices $A_{1}$ and $A_{2}$ such that $A=\left[A_{1}, A_{2}\right]$ and $\operatorname{tr}\left(B A_{1}\right)=\operatorname{tr}\left(B A_{2}\right)=0$, then we also have $\operatorname{tr}\left((B-\lambda A) A_{1}\right)=$ $\operatorname{tr}\left((B-\lambda A) A_{2}\right)=0$. Indeed, equality $A=\left[A_{1}, A_{2}\right]$ ensures that $\operatorname{tr}\left(A A_{1}\right)=$ $\operatorname{tr}\left(A A_{2}\right)=0$ (see Lemma 1.6).

(3) Let $(A, B) \in \mathrm{M}_{n}(\mathbb{K})^{2}$ and $P \in \mathrm{GL}_{n}(\mathbb{K})$. Setting $\mathcal{G}:=\{B\}^{\perp}$, we see that the assumption $A \in[\mathcal{G}, \mathcal{G}]$ implies $P A P^{-1} \in\left[P \mathcal{G} P^{-1}, P \mathcal{G} P^{-1}\right]$, while $P \mathcal{G} P^{-1}=$ $\left\{P B P^{-1}\right\}^{\perp}$.

Now, let us give a rough idea of the proof strategy. One fixes $A \in \mathfrak{s l}_{n}(\mathbb{K})$ and aims at proving that $A \in[\mathcal{H}, \mathcal{H}]$. We fix a non-zero matrix $B$ such that $\mathcal{H}=\{B\}^{\perp}$.

Our basic strategy is the Albert-Muckenhoupt method: We try to find a cyclic matrix $M$ in $\mathcal{H}$ such that $A \in \operatorname{im}\left(\operatorname{ad}_{M}\right)$; if $A \notin \operatorname{ad}_{M}(\mathcal{H})$, then we learn that $\mathcal{C}(M) \subset \mathcal{H}$ (see principle (1) above), which yields additional information on $B$. Most of the time, we will search for such a cyclic matrix $M$ among the nilpotent matrices with rank $n-1$. The most favorable situation is the one where $A$ is either upper-triangular or Hessenberg with enough non-zero sub-diagonal entries: In these cases, we search for a good matrix $M$ among the strictly upper-triangular matrices with rank $n-1$ (see Lemma 2.2). If this method yields no solution, then we learn precious information on the simultaneous reduction of the endomorphisms $X \mapsto A X$ and $X \mapsto B X$. Using changes of bases, we shall see that either the above method delivers a solution for a pair $\left(A^{\prime}, B^{\prime}\right)$ that is simultaneously similar to $(A, B)$, in which case Principle $(3)$ shows that we have a solution for $(A, B)$, or $\left(I_{n}, A, B\right)$ is locally linearly dependent (see the definition below), or else $n=3$ and $A$ is similar to $\lambda I_{3}+E_{2,3}$ for some $\lambda \in \mathbb{K}$. When $\left(I_{n}, A, B\right)$ is locally linearly dependent and $A$ is not of that special type, one uses the classification of locally linearly dependent triples to reduce the situation to the one where $B=I_{n}$, that is $\mathcal{H}=\mathfrak{s l}_{n}(\mathbb{K})$, and in that case the proof is completed by invoking Theorem 1.1. Finally, the case when $A$ is similar to $\lambda I_{3}+E_{2,3}$ for some $\lambda \in \mathbb{K}$ will be dealt with independently (Section 2.5) by applying Albert and Muckenhoupt's method for well-chosen companion matrices instead of a Jordan nilpotent matrix.

Let us finish these strategic considerations by recalling the notion of local linear dependence:

Definition 2.1. Given vector spaces $U$ and $V$, linear maps $f_{1}, \ldots, f_{n}$ from $U$ to $V$ are called locally linearly dependent (in abbreviated form: $L L D$ ) when the vectors 
$f_{1}(x), \ldots, f_{n}(x)$ are linearly dependent for all $x \in U$.

We adopt a similar definition for matrices by referring to the linear maps that are canonically associated with these matrices.

\subsection{The basic lemma.}

Lemma 2.2. Let $(A, B) \in \mathfrak{s l}_{n}(\mathbb{K})^{2}$ be with $B=\left(b_{i, j}\right) \neq 0$, and set $\mathcal{H}:=\{B\}^{\perp}$. In each one of the following cases, $A$ belongs to $[\mathcal{H}, \mathcal{H}]$ :

(a) $\# \mathbb{K}>2, A$ is upper-triangular and $B$ is not Hessenberg.

(b) $\# \mathbb{K}>3, A$ is Hessenberg and there exist $i \in \llbracket 2, n-1 \rrbracket$ and $j \in \llbracket 3, n \rrbracket \backslash\{i\}$ such that $\{1, i\} \subset \ell(A)$ and $b_{j, 1} \neq 0$.

Proof. We use a reductio ad absurdum, assuming that $A \notin[\mathcal{H}, \mathcal{H}]$. We write $A=\left(a_{i, j}\right)$.

(a) Assume that $\# \mathbb{K}>2$, that $A$ is upper-triangular and that $B$ is not Hessenberg. We choose a pair $\left(l, l^{\prime}\right) \in \llbracket 1, n \rrbracket^{2}$ such that $b_{l, l^{\prime}} \neq 0$, with $l-l^{\prime}$ maximal for such pairs. Thus, $l-l^{\prime}>1$. Let $\left(x_{1}, \ldots, x_{n-1}\right) \in\left(\mathbb{K}^{*}\right)^{n-1}$, and set

$$
\beta:=\frac{\sum_{k=1}^{n-1} b_{k+1, k} x_{k}}{b_{l, l^{\prime}}} \text { and } \quad M:=\sum_{k=1}^{n-1} x_{k} E_{k, k+1}-\beta E_{l^{\prime}, l} .
$$

We see that $M$ is nilpotent of rank $n-1$, and hence, it is cyclic. One notes that $M \in \mathcal{H}$. Moreover, $\operatorname{tr}\left(A M^{k}\right)=0$ for all $k \geq 1$, because $A$ is upper-triangular and $M$ is strictly upper-triangular, whereas $\operatorname{tr}(A)=0$ by assumption. Thus, $A \in \operatorname{im}\left(\operatorname{ad}_{M}\right)$. As it is assumed that $A \notin \operatorname{ad}_{M}(\mathcal{H})$, one deduces from principle (1) in Section 2.1 that $\mathcal{C}(M) \subset \mathcal{H}$; in particular $\operatorname{tr}\left(M^{l-l^{\prime}} B\right)=0$, which, as $b_{i, j}=0$ whenever $i-j>l-l^{\prime}$, reads

$$
b_{l-l^{\prime}+1,1} x_{1} x_{2} \cdots x_{l-l^{\prime}}+b_{l-l^{\prime}+2,2} x_{2} x_{3} \cdots x_{l-l^{\prime}+1}+\cdots+b_{n, n-l+l^{\prime}} x_{n-l+l^{\prime}} \cdots x_{n-1}=0 .
$$

Here, we have a polynomial with degree at most 1 in each variable $x_{i}$, and this polynomial vanishes at every $\left(x_{1}, \ldots, x_{n-1}\right) \in\left(\mathbb{K}^{*}\right)^{n-1}$, with $\# \mathbb{K}^{*} \geq 2$. It follows that $b_{i, j}=0$ for all $(i, j) \in \llbracket 1, n \rrbracket^{2}$ with $i-j=l-l^{\prime}$, and the special case $(i, j)=\left(l, l^{\prime}\right)$ yields a contradiction.

(b) Now, we assume that $\# \mathbb{K}>3$, that $A$ is Hessenberg and that there exist $i \in \llbracket 2, n \rrbracket$ and $j \in \llbracket 3, n \rrbracket \backslash\{i\}$ such that $\{1, i\} \subset \ell(A)$ and $b_{j, 1} \neq 0$. The proof strategy is similar to the one of case (a), with additional technicalities. One chooses a pair $\left(l, l^{\prime}\right) \in \llbracket 1, n \rrbracket^{2}$ such that $b_{l, l^{\prime}} \neq 0$, with $l-l^{\prime}$ maximal for such pairs (again, the assumptions yield $l-l^{\prime} \geq j-1>1$ ). As $a_{2,1} \neq 0$, no generality is lost in assuming that $a_{2,1}=1$. We introduce the formal polynomial

$$
\mathbf{p}:=\sum_{k=1}^{n-2} a_{k+2, k+1} \mathbf{x}_{k} \in \mathbb{K}\left[\mathbf{x}_{1}, \mathbf{x}_{2}, \ldots, \mathbf{x}_{n-2}\right]
$$


Let $\left(x_{1}, \ldots, x_{n-2}\right) \in\left(\mathbb{K}^{*}\right)^{n-2}$, and set

$$
\alpha:=\mathbf{p}\left(x_{1}, \ldots, x_{n-2}\right) \quad \text { and } \quad \beta:=\frac{\alpha b_{2,1}-\sum_{k=1}^{n-2} x_{k} b_{k+2, k+1}}{b_{l, l^{\prime}}} .
$$

Finally, set

$$
M:=-\alpha E_{1,2}+\sum_{k=1}^{n-2} x_{k} E_{k+1, k+2}+\beta E_{l^{\prime}, l} .
$$

The definition of $M$ shows that $\operatorname{tr}(M A)=\operatorname{tr}(M B)=0$, and in particular $M \in \mathcal{H}$. Assume now that $\mathbf{p}\left(x_{1}, \ldots, x_{n-2}\right) \neq 0$. Then, $M$ is cyclic as it is nilpotent with rank $n-1$. As $A$ is Hessenberg, we also see that $\operatorname{tr}\left(M^{k} A\right)=0$ for all $k \geq 2$. Thus, $\operatorname{tr}\left(M^{k} A\right)=0$ for every non-negative integer $k$, and hence, Lemma 1.7 yields $A \in \operatorname{im}\left(\operatorname{ad}_{M}\right)$. It ensues that $\mathcal{C}(M) \subset \mathcal{H}$, and in particular $\operatorname{tr}\left(M^{j-1} B\right)=0$. As $l-l^{\prime}>1$, we see that, for all $(a, b) \in \llbracket 1, n \rrbracket^{2}$ with $b-a \leq l-l^{\prime}$, and every integer $c>1$, the matrices $M^{c}$ and $\left(-\alpha E_{1,2}+\sum_{k=1}^{n-2} x_{k} E_{k+1, k+2}\right)^{c}$ have the same entry at the $(a, b)$-spot; in particular, for all $k \in \llbracket 2, n-j+1 \rrbracket$, the entry of $M^{j-1}$ at the $(k, j+k-1)$-spot is $x_{k-1} x_{k} \cdots x_{k-3+j}$, and the entry of $M^{j-1}$ at the $(1, j)$-spot is $-\alpha x_{1} \cdots x_{j-2}$; moreover, for all $(a, b) \in \llbracket 1, n \rrbracket^{2}$ with $b-a \leq \ell-\ell^{\prime}$ and $b-a \neq j-1$, the entry of $M^{j-1}$ at the $(a, b)$-spot is 0 . Therefore, equality $\operatorname{tr}\left(M^{j-1} B\right)=0$ yields

$-b_{j, 1} \alpha x_{1} \cdots x_{j-2}+b_{j+1,2} x_{1} \cdots x_{j-1}+b_{j+2,3} x_{2} \cdots x_{j}+\cdots+b_{n, n-j+1} x_{n-j} \cdots x_{n-2}=0$.

We conclude that we have established the following identity: For the polynomial

$$
\mathbf{q}:=\mathbf{p} \times\left(-b_{j, 1} \mathbf{p} \mathbf{x}_{1} \cdots \mathbf{x}_{j-2}+b_{j+1,2} \mathbf{x}_{1} \cdots \mathbf{x}_{j-1}+\cdots+b_{n, n-j+1} \mathbf{x}_{n-j} \cdots \mathbf{x}_{n-2}\right),
$$

we have

$$
\forall\left(x_{1}, \ldots, x_{n-2}\right) \in\left(\mathbb{K}^{*}\right)^{n-2}, \quad \mathbf{q}\left(x_{1}, \ldots, x_{n-2}\right)=0
$$

Noting that $\mathbf{q}$ has degree at most 3 in each variable, we split the discussion into two main cases.

Case 1. \#KK $>4$.

Then, $\# \mathbb{K}^{*}>3$ and hence $\mathbf{q}=0$. As $\mathbf{p} \neq 0$ (remember that $a_{i+1, i} \neq 0$ ), it follows that

$-b_{j, 1} \mathbf{p} \mathbf{x}_{1} \cdots \mathbf{x}_{j-2}+b_{j+1,2} \mathbf{x}_{1} \cdots \mathbf{x}_{j-1}+b_{j+2,3} \mathbf{x}_{2} \cdots \mathbf{x}_{j}+\cdots+b_{n, n-j+1} \mathbf{x}_{n-j} \cdots \mathbf{x}_{n-2}=0$.

As $b_{j, 1} \neq 0$, identifying the coefficients of the monomials of type $\mathbf{x}_{1} \cdots \mathbf{x}_{j-2} \mathbf{x}_{k}$ with $k \in \llbracket 1, n-2 \rrbracket \backslash\{j-1\}$ leads to $a_{k+2, k+1}=0$ for all such $k$. This contradicts the assumption that $a_{i+1, i} \neq 0$. 
Case 2. $\# \mathbb{K}=4$.

A polynomial of $\mathbb{K}[t]$ which vanishes at every non-zero element of $\mathbb{K}$ must be a multiple of $t^{3}-1$. In particular, if such a polynomial has degree at most 3 , we may write it as $\alpha_{3} t^{3}+\alpha_{2} t^{2}+\alpha_{1} t+\alpha_{0}$, and we obtain $\alpha_{3}=-\alpha_{0}$. From there, we split the discussion into two subcases.

Subcase 2.1. $i>j$.

Then, $\mathbf{q}$ has degree at most 2 in $\mathbf{x}_{i-1}$. Thus, if we see $\mathbf{q}$ as a polynomial in the sole variable $\mathbf{x}_{i-1}$, the coefficients of this polynomial must vanish for every specialization of $\mathbf{x}_{1}, \ldots, \mathbf{x}_{i-2}, \mathbf{x}_{i}, \ldots, \mathbf{x}_{n-2}$ in $\mathbb{K}^{*}$; extracting the coefficients of $\left(\mathbf{x}_{i-1}\right)^{2}$ leads to the identity

$\forall\left(x_{1}, \ldots, x_{i-2}, x_{i}, \ldots, x_{n-2}\right) \in\left(\mathbb{K}^{*}\right)^{n-3}, \quad-b_{j, 1}\left(a_{i+1, i}\right)^{2} x_{1} \cdots x_{j-2}+\mathbf{r}\left(x_{1}, \ldots, x_{n-2}\right)=0$, where $\mathbf{r}=\sum_{k=i-j+1}^{n-j} a_{i+1, i} b_{j+k, k+1} \mathbf{x}_{k} \cdots \mathbf{x}_{i-2} \mathbf{x}_{i} \cdots \mathbf{x}_{j-2+k}$. Noting that the degree of $-b_{j, 1}\left(a_{i+1, i}\right)^{2} \mathbf{x}_{1} \cdots \mathbf{x}_{j-2}+\mathbf{r}$ is at most 1 in each variable, we deduce that this polynomial is zero. This contradicts the fact that the coefficient of $\mathbf{x}_{1} \cdots \mathbf{x}_{j-2}$ is $-b_{j, 1}\left(a_{i+1, i}\right)^{2}$, which is non-zero according to our assumptions.

Subcase 2.2. $i<j$.

Let us fix $x_{1}, \ldots, x_{i-2}, x_{i}, \ldots, x_{n-2}$ in $\mathbb{K}^{*}$. The coefficient of $\mathbf{q}\left(x_{1}, \ldots, x_{i-2}, \mathbf{x}_{i-1}\right.$, $\left.x_{i}, \ldots, x_{n-2}\right)$ with respect to $\left(\mathbf{x}_{i-1}\right)^{3}$ is

$$
-b_{j, 1}\left(a_{i+1, i}\right)^{2} x_{1} \cdots x_{i-2} x_{i} \cdots x_{j-2} .
$$

One the other hand, with

$$
\mathbf{s}:=\sum_{i \leq k \leq n-j} b_{j+k, k+1} \prod_{\ell=k}^{j-2+k} \mathbf{x}_{\ell}
$$

the coefficient of $\mathbf{q}\left(x_{1}, \ldots, x_{i-2}, \mathbf{x}_{i-1}, x_{i}, \ldots, x_{n-2}\right)$ with respect to $\left(\mathbf{x}_{i-1}\right)^{0}$ is

$$
\mathbf{s}\left(x_{1}, \ldots, x_{i-2}, x_{i}, \ldots, x_{n-2}\right) \sum_{k \in \llbracket 1, n-2 \rrbracket \backslash\{i-1\}} a_{k+2, k+1} x_{k} .
$$

Therefore, for all $\left(x_{1}, \ldots, x_{n-2}\right) \in\left(\mathbb{K}^{*}\right)^{n-2}$,

$$
\begin{aligned}
b_{j, 1}\left(a_{i+1, i}\right)^{2} x_{1} \cdots x_{i-2} x_{i} \cdots x_{j-2}= & \mathbf{s}\left(x_{1}, \ldots, x_{i-2}, x_{i}, \ldots, x_{n-2}\right) \\
& \times \sum_{k \in \llbracket 1, n-2 \rrbracket \backslash\{i-1\}} a_{k+2, k+1} x_{k} .
\end{aligned}
$$

On both sides of this equality, we have polynomials of degree at most 2 in each variable. As $\#\left(\mathbb{K}^{*}\right)>2$, we deduce the identity

$$
b_{j, 1}\left(a_{i+1, i}\right)^{2} \mathbf{x}_{1} \ldots \mathbf{x}_{i-2} \mathbf{x}_{i} \cdots \mathbf{x}_{j-2}=\mathbf{s} \times \sum_{k \in \llbracket 1, n-2 \rrbracket \backslash\{i-1\}} a_{k+2, k+1} \mathbf{x}_{k} .
$$

However, on the left-hand side of this identity is a non-zero homogeneous polynomial of degree $j-3$, whereas its right-hand side is a homogeneous polynomial of degree $j$. There lies a final contradiction. 
2.3. Reduction to the case when $I_{n}, A, B$ are locally linearly dependent. In this section, we use Lemma 2.2 to prove the following result:

Lemma 2.3. Assume that $\# \mathbb{K}>3$, let $(A, B) \in \mathfrak{s l}_{n}(\mathbb{K})^{2}$ be such that $B \neq 0$, and set $\mathcal{H}:=\{B\}^{\perp}$. Then, either $A \in[\mathcal{H}, \mathcal{H}]$, or $\left(I_{n}, A, B\right)$ is LLD, or $A$ is similar to $\lambda I_{3}+E_{2,3}$ for some $\lambda \in \mathbb{K}$.

In order to prove Lemma 2.3. one needs two preliminary results. The first one is a basic result in the theory of matrix spaces with rank bounded above.

Lemma 2.4 (Lemma 2.4 of [6]). Let $m, n, p, q$ be positive integers, and $\mathcal{V}$ be a linear subspace of $\mathrm{M}_{m+p, n+q}(\mathbb{K})$ in which every matrix splits up as

$$
M=\left[\begin{array}{cc}
A(M) & ? \\
0 & B(M)
\end{array}\right],
$$

where $A(M) \in \mathrm{M}_{m, n}(\mathbb{K})$ and $B(M) \in \mathrm{M}_{p, q}(\mathbb{K})$. Assume that there is an integer $r$ such that $\forall M \in \mathcal{V}, \operatorname{rk}(M) \leq r<\# \mathbb{K}$, and set $s:=\max \{\operatorname{rk}(A(M)) \mid M \in \mathcal{V}\}$ and $t:=\max \{\operatorname{rk}(B(M)) \mid M \in \mathcal{V}\}$. Then, $s+t \leq r$.

Lemma 2.5. Assume that $\# \mathbb{K} \geq 3$. Let $V$ be a vector space over $\mathbb{K}$ and $u$ be an endomorphism of $V$ that is not a scalar multiple of the identity. Then, there are two linearly independent non-eigenvectors of $u$.

Proof. As $u$ is not a scalar multiple of the identity, some vector $x \in V \backslash\{0\}$ is not an eigenvector of $u$. Then, the 2 -dimensional subspace $P:=\operatorname{span}(x, u(x))$ contains $x$. As $u_{\mid P}$ is not a scalar multiple of the identity, $u$ stabilizes at most two 1-dimensional subspaces of $P$. As $\# \mathbb{K}>2$, there are at least four 1-dimensional subspaces of $P$, whence at least two of them are not stable under $u$. This proves our claim.

Now, we are ready to prove Lemma 2.3 .

Proof of Lemma 2.3. Throughout the proof, we assume that $A \notin[\mathcal{H}, \mathcal{H}]$ and that there is no scalar $\lambda$ such that $A$ is similar to $\lambda I_{3}+E_{2,3}$. Our aim is to show that $\left(I_{n}, A, B\right)$ is LLD.

Note that, for all $P \in \mathrm{GL}_{n}(\mathbb{K})$, no pair $(M, N) \in \mathrm{M}_{n}(\mathbb{K})^{2}$ satisfies both $[M, N]=$ $P^{-1} A P$ and $\operatorname{tr}\left(\left(P^{-1} B P\right) M\right)=\operatorname{tr}\left(\left(P^{-1} B P\right) N\right)=0$.

Let us say that a vector $x \in \mathbb{K}^{n}$ has order 3 when $\operatorname{rk}\left(x, A x, A^{2} x\right)=3$. Let $x \in \mathbb{K}^{n}$ be of order 3 . Then, $\left(x, A x, A^{2} x\right)$ may be extended into a basis $\mathbf{B}=$ $\left(x_{1}, x_{2}, x_{3}, x_{4}, \ldots, x_{n}\right)$ of $\mathbb{K}^{n}$ such that $A^{\prime}:=P_{\mathbf{B}}^{-1} A P_{\mathbf{B}}$ is Hessenberg 1 . Moreover, one sees that $\{1,2\} \subset \ell\left(A^{\prime}\right)$. Applying point (a) of Lemma 2.2, one obtains that the

\footnotetext{
${ }^{1}$ One finds such a basis by induction as follows: One sets $\left(x_{1}, x_{2}, x_{3}\right):=\left(x, A x, A^{2} x\right)$ and, given $k \in \llbracket 4, n \rrbracket$ such that $x_{1}, \ldots, x_{k-1}$ are defined, one sets $x_{k}:=A x_{k-1}$ if $A x_{k-1} \notin \operatorname{span}\left(x_{1}, \ldots, x_{k-1}\right)$, otherwise one chooses an arbitrary vector $x_{k} \in \mathbb{K}^{n} \backslash \operatorname{span}\left(x_{1}, \ldots, x_{k-1}\right)$.
} 
entries in the first column of $P_{\mathbf{B}}^{-1} B P_{\mathbf{B}}$ are all zero starting from the third one, which means that $B x \in \operatorname{span}(x, A x)$.

Let now $x \in \mathbb{K}^{n}$ be a vector that is not of order 3 . If $x$ and $A x$ are linearly dependent, then $x, A x, B x$ are linearly dependent. Thus, we may assume that $\operatorname{rk}(x, A x)=2$ and $A^{2} x \in \operatorname{span}(x, A x)$. We split $\mathbb{K}^{n}=\operatorname{span}(x, A x) \oplus F$ and we choose a basis $\left(f_{3}, \ldots, f_{n}\right)$ of $F$. For $\mathbf{B}:=\left(x, A x, f_{3}, \ldots, f_{n}\right)$, we now have, for some $(\alpha, \beta) \in \mathbb{K}^{2}$ and some $N \in \mathrm{M}_{n-2}(\mathbb{K})$,

$$
P_{\mathbf{B}}^{-1} A P_{\mathbf{B}}=\left[\begin{array}{cc}
K & ? \\
0 & N
\end{array}\right], \quad \text { where } K=\left[\begin{array}{cc}
0 & \alpha \\
1 & \beta
\end{array}\right] .
$$

From there, we split the discussion into several cases, depending on the form of $N$ and its relationship with $K$.

Case 1. $N \notin \mathbb{K} I_{n-2}$.

Then, there is a vector $y \in \mathbb{K}^{n-2}$ for which $y$ and $N y$ are linearly independent. Denoting by $z$ the vector of $F$ with coordinate list $y$ in $\left(f_{3}, \ldots, f_{n}\right)$, one obtains $\operatorname{rk}(x, A x, z, A z)=4$, and hence, one may extend $(x, A x, z, A z)$ into a basis $\mathbf{B}^{\prime}$ of $\mathbb{K}^{n}$ such that $A^{\prime}:=P_{\mathbf{B}^{\prime}}^{-1} A P_{\mathbf{B}^{\prime}}$ is Hessenberg with $\{1,3\} \subset \ell\left(A^{\prime}\right)$. Point (b) of Lemma 2.2 shows that, in the first column of $P_{\mathbf{B}^{\prime}}^{-1} B P_{\mathbf{B}^{\prime}}$, all the entries must be zero starting from the fourth one, yielding $B x \in \operatorname{span}(x, A x, z)$. As $N \notin \mathbb{K} I_{n-2}$, we know from Lemma 2.5 that we may find another vector $z^{\prime} \in F \backslash \mathbb{K} z$ such that $\operatorname{rk}\left(x, A x, z^{\prime}, A z^{\prime}\right)=4$, which yields $B x \in \operatorname{span}\left(x, A x, z^{\prime}\right)$. Thus, $B x \in \operatorname{span}(x, A x, z) \cap \operatorname{span}\left(x, A x, z^{\prime}\right)=$ $\operatorname{span}(x, A x)$.

Case 2. $N=\lambda I_{n-2}$ for some $\lambda \in \mathbb{K}$.

Subcase 2.1. $\lambda$ is not an eigenvalue of $K$.

Then, $G:=\operatorname{Ker}\left(A-\lambda I_{n}\right)$ has dimension $n-2$. For $z \in \mathbb{K}^{n}$, denote by $p_{z}$ the monic generator of the ideal $\{q \in \mathbb{K}[t]: q(A) z=0\}$. Recall that, given $y$ and $z$ in $\mathbb{K}^{n}$ for which $p_{y}$ and $p_{z}$ are mutually prime, one has $p_{y+z}=p_{y} p_{z}$. In particular, as $p_{x}$ has degree $2, p_{z}$ has degree 3 for every $z \in(\mathbb{K} x \oplus G) \backslash(\mathbb{K} x \cup G)$, that is every $z$ in $(\mathbb{K} x \oplus G) \backslash(\mathbb{K} x \cup G)$ has order 3 ; thus, $\operatorname{rk}(z, A z, B z) \leq 2$ for all such $z$. Moreover, it is obvious that $\operatorname{rk}(z, A z, B z) \leq 2$ for all $z \in G$.

Let us choose a non-zero linear form $\varphi$ on $\mathbb{K} x \oplus G$ such that $\varphi(x)=0$. For every $z \in \mathbb{K} x \oplus G$, set

$$
M(z)=\left[\begin{array}{cccc}
\varphi(z) & 0 & 0 & 0 \\
0 & z & A z & B z
\end{array}\right] \in \mathrm{M}_{n+1,4}(\mathbb{K}) .
$$

Then, with the above results, we know that $\operatorname{rk}(M(z)) \leq 3$ for all $z \in \mathbb{K} x \oplus G$. On the other hand, $\max \{\operatorname{rk}(\varphi(z)) \mid z \in(\mathbb{K} x \oplus G)\}=1$. Using Lemma 2.4 we deduce that $\operatorname{rk}(z, A z, B z) \leq 2$ for all $z \in \mathbb{K} x \oplus G$. In particular, $\operatorname{rk}(x, A x, B x) \leq 2$. 
Subcase 2.2. $\lambda$ is an eigenvalue of $K$ with multiplicity 1 .

Then, there are eigenvectors $y$ and $z$ of $A$, with distinct corresponding eigenvalues, such that $x=y+z$. Thus, $(y, z)$ may be extended into a basis $\mathbf{B}^{\prime}$ of $\mathbb{K}^{n}$ such that $P_{\mathbf{B}^{\prime}}^{-1} A P_{\mathbf{B}^{\prime}}$ is upper-triangular. It follows from point (a) of Lemma 2.2 that $P_{\mathbf{B}^{\prime}}^{-1} B P_{\mathbf{B}^{\prime}}$ is Hessenberg, and in particular $B y \in \operatorname{span}(y, z)$. Starting from $(z, y)$ instead of $(y, z)$, one finds $B z \in \operatorname{span}(y, z)$. Therefore, all the vectors $y+z, A(y+z)$ and $B(y+z)$ belong to the 2 -dimensional $\operatorname{space} \operatorname{span}(y, z)$, which yields $\operatorname{rk}(x, A x, B x) \leq 2$.

Subcase 2.3. $\lambda$ is an eigenvalue of $K$ with multiplicity 2 .

Then, the characteristic polynomial of $A$ is $(t-\lambda)^{n}$.

- Assume that $n \geq 4$. One chooses an eigenvector $y$ of $A$ in $\operatorname{span}(x, A x)$, so that $(y, x)$ is a basis of $\operatorname{span}(x, A x)$. Then, one chooses an arbitrary nonzero vector $u \in F$, and one extends $(y, x, u)$ into a basis $\mathbf{B}^{\prime}$ of $\mathbb{K}^{n}$ such that $P_{\mathbf{B}^{\prime}}^{-1} A P_{\mathbf{B}^{\prime}}$ is upper-triangular. Applying point (a) of Lemma 2.2 once more yields $B x \in \operatorname{span}(y, x, u)=\operatorname{span}(x, A x, u)$. As $n \geq 4$, we can choose another vector $v \in F \backslash \mathbb{K} u$, and the above method yields $B x \in \operatorname{span}(x, A x, v)$, while $x, A x, u, v$ are linearly independent. Therefore, $B x \in \operatorname{span}(x, A x, u) \cap$ $\operatorname{span}(x, A x, v)=\operatorname{span}(x, A x)$.

- Finally, assume that $n=3$. As $A$ is not similar to $\lambda I_{3}+E_{2,3}$, the only remaining option is that $\operatorname{rk}\left(A-\lambda I_{3}\right)=2$. Then, we can find a linear form $\varphi$ on $\mathbb{K}^{3}$ with kernel $\operatorname{Ker}\left(A-\lambda I_{3}\right)^{2}$. Every vector $z \in \mathbb{K}^{3} \backslash \operatorname{Ker}\left(A-\lambda I_{3}\right)^{2}$ has order 3. Therefore, for every $z \in \mathbb{K}^{3}$, either $\varphi(z)=0$ or $\operatorname{rk}(z, A z, B z) \leq 2$. With the same line of reasoning as in Subcase 2.1, we obtain $\operatorname{rk}(x, A x, B x) \leq 2$. This completes the proof.

Thus, only two situations are left to consider: The one where $\left(I_{n}, A, B\right)$ is LLD, and the one where $A$ is similar to $\lambda I_{3}+E_{2,3}$ for some $\lambda \in \mathbb{K}$. They are dealt with separately in the next two sections.

2.4. The case when $\left(I_{n}, A, B\right)$ is locally linearly dependent. In order to analyze the situation where $\left(I_{n}, A, B\right)$ is LLD, we use the classification of LLD triples over fields with more than 2 elements (this result is found in [7]; prior to that, the result was known for infinite fields [2] and for fields with more than 4 elements [3]).

Theorem 2.6 (Classification theorem for LLD triples). Let $(f, g, h)$ be an $L L D$ triple of linear operators from a vector space $U$ to a vector space $V$, where the underlying field has more than 2 elements. Assume that $f, g, h$ are linearly independent and that $\operatorname{Ker}(f) \cap \operatorname{Ker}(g) \cap \operatorname{Ker}(h)=\{0\}$ and $\operatorname{im}(f)+\operatorname{im}(g)+\operatorname{im}(h)=V$. Then:

(a) Either there is a 2-dimensional subspace $\mathcal{P}$ of $\operatorname{span}(f, g, h)$ and a 1-dimensional subspace $\mathcal{D}$ of $V$ such that $\operatorname{im}(u) \subset \mathcal{D}$ for all $u \in \mathcal{P}$;

(b) $\operatorname{Or} \operatorname{dim} V \leq 2$; 
(c) $\operatorname{Or} \operatorname{dim} U=\operatorname{dim} V=3$ and there are bases of $U$ and $V$ in which the operator space $\operatorname{span}(f, g, h)$ is represented by the space $\mathrm{A}_{3}(\mathbb{K})$ of all $3 \times 3$ alternating matrices.

Corollary 2.7. Assume that \#K $>2$, and let $A$ and $B$ be matrices of $\mathrm{M}_{n}(\mathbb{K})$, with $n \geq 3$, such that $\left(I_{n}, A, B\right)$ is $L L D$. Then, either $I_{n}, A, B$ are linearly dependent, or there is a 1-dimensional subspace $\mathcal{D}$ of $\mathbb{K}^{n}$ and scalars $\lambda$ and $\mu$ such that $\operatorname{im}(A-$ $\left.\lambda I_{n}\right)=\mathcal{D}=\operatorname{im}\left(B-\mu I_{n}\right)$.

Proof. Assume that $I_{n}, A, B$ are linearly independent. As $\operatorname{Ker} I_{n}=\{0\}$ and $\operatorname{im} I_{n}=\mathbb{K}^{n}$, we are in the position to use Theorem 2.6. Moreover, $\operatorname{rk} I_{n}>2$ discards Cases (b) and (c) altogether (as no $3 \times 3$ alternating matrix is invertible). Therefore, we have a 2-dimensional subspace $\mathcal{P}$ of $\operatorname{span}\left(I_{n}, A, B\right)$ and a 1 -dimensional subspace $\mathcal{D}$ of $\mathbb{K}^{n}$ such that $\operatorname{im} M \subset \mathcal{D}$ for all $M \in \mathcal{P}$. In particular $I_{n} \notin \mathcal{P}$, whence $\operatorname{span}\left(I_{n}, A, B\right)=$ $\mathbb{K} I_{n} \oplus \mathcal{P}$. This yields a pair $\left(\lambda, M_{1}\right) \in \mathbb{K} \times \mathcal{P}$ such that $A=\lambda I_{n}+M_{1}$, and hence, $\operatorname{im}\left(A-\lambda I_{n}\right) \subset \mathcal{D}$. As $A-\lambda I_{n} \neq 0$ (we have assumed that $I_{n}, A, B$ are linearly independent), we deduce that $\operatorname{im}\left(A-\lambda I_{n}\right)=\mathcal{D}$. Similarly, one finds a scalar $\mu$ such that $\operatorname{im}\left(B-\mu I_{n}\right)=\mathcal{D}$.

From there, we can prove the following result as a consequence of Theorem 1.1

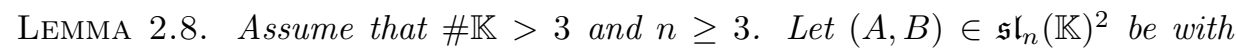
$B \neq 0$, and set $\mathcal{H}:=\{B\}^{\perp}$. Assume that $\left(I_{n}, A, B\right)$ is LLD and that $A$ is not similar to $\lambda I_{3}+E_{2,3}$ for some $\lambda \in \mathbb{K}$. Then, $A \in[\mathcal{H}, \mathcal{H}]$.

Proof. We use a reductio ad absurdum by assuming that $A \notin[\mathcal{H}, \mathcal{H}]$. By Corollary 2.7. we can split the discussion into two main cases.

Case 1. $I_{n}, A, B$ are linearly dependent.

Assume first that $A \in \mathbb{K} I_{n}$. Then, $P^{-1} A P$ is upper-triangular for every $P \in \mathrm{GL}_{n}(\mathbb{K})$, and hence, Lemma 2.2 yields that $P^{-1} B P$ is Hessenberg for every such $P$. In particular, let $x \in \mathbb{K}^{n} \backslash\{0\}$. For every $y \in \mathbb{K}^{n} \backslash \mathbb{K} x$, we can extend $(x, y)$ into a basis $\left(x, y, y_{3}, \ldots, y_{n}\right)$ of $\mathbb{K}^{n}$, and hence, we learn that $B x \in \operatorname{span}(x, y)$. Using the basis $\left(x, y_{3}, y, y_{4}, \ldots, y_{n}\right)$, we also find $B x \in \operatorname{span}\left(x, y_{3}\right)$, whence $B x \in \mathbb{K} x$. Varying $x$, we deduce that $B \in \mathbb{K} I_{n}$, whence $\mathcal{H}=\mathfrak{s l}_{n}(\mathbb{K})$. Theorem 1.1 then yields $A \in[\mathcal{H}, \mathcal{H}]$, contradicting our assumptions.

Assume now that $A \notin \mathbb{K} I_{n}$. Then, there are scalars $\lambda$ and $\mu$ such that $B=\lambda A+$ $\mu I_{n}$. By Theorem 1.1 there are trace zero matrices $M$ and $N$ such that $A=[M, N]$. Thus, $\operatorname{tr}((B-\lambda A) M)=\operatorname{tr}((B-\lambda A) N)=0$. Using principle (2) of Section 2.1, we deduce that $(M, N) \in \mathcal{H}^{2}$, whence $A \in[\mathcal{H}, \mathcal{H}]$.

Case 2. $I_{n}, A, B$ are linearly independent.

By Corollary 2.7, there are scalars $\lambda$ and $\mu$ together with a 1-dimensional subspace $\mathcal{D}$ of $\mathbb{K}^{n}$ such that $\operatorname{im}\left(A-\lambda I_{n}\right)=\operatorname{im}\left(B-\mu I_{n}\right)=\mathcal{D}$. In particular, $A-\lambda I_{n}$ has rank 
1 , and hence, it is diagonalisable or nilpotent. In any case, $A$ is triangularizable; in the second case, the assumption that $A$ is not similar to $\lambda I_{3}+E_{2,3}$ leads to $n \geq 4$.

Let $x$ be an eigenvector of $A$. Then, we can extend $x$ into a triple $(x, y, z)$ of linearly independent eigenvectors of $A$ (this uses $n \geq 4$ in the case when $A-\lambda I_{n}$ is nilpotent). Then, we further extend this triple into a basis $\left(x, y, z, y_{4}, \ldots, y_{n}\right)$ in which $v \mapsto A v$ is upper-triangular. Point (a) in Lemma 2.2 yields $B x \in \operatorname{span}(x, y)$. With the same line of reasoning, $B x \in \operatorname{span}(x, z)$, and hence, $B x \in \operatorname{span}(x, y) \cap \operatorname{span}(x, z)=\mathbb{K} x$. Thus, we have proved that every eigenvector of $A$ is an eigenvector of $B$. In particular, $\operatorname{Ker}\left(A-\lambda I_{n}\right)$ is stable under $v \mapsto B v$, and the resulting endomorphism is a scalar multiple of the identity. This provides us with some $\alpha \in \mathbb{K}$ such that $\left(B-\alpha I_{n}\right) z=0$ for all $z \in \operatorname{Ker}\left(A-\lambda I_{n}\right)$. In particular, $\alpha$ is an eigenvalue of $B$ with multiplicity at least $n-1$, and since $\mu$ shares this property and $n<2(n-1)$, we deduce that $\alpha=\mu$. As $\operatorname{rk}\left(A-\lambda I_{n}\right)=\operatorname{rk}\left(B-\mu I_{n}\right)=1$, we deduce that $\operatorname{Ker}\left(A-\lambda I_{n}\right)=\operatorname{Ker}\left(B-\mu I_{n}\right)$. Thus, $A-\lambda I_{n}$ and $B-\mu I_{n}$ are two rank 1 matrices with the same kernel and the same range, and hence, they are linearly dependent. This contradicts the assumption that $I_{n}, A, B$ be linearly independent, thereby completing the proof.

\subsection{The case when $A=\lambda I_{3}+E_{2,3}$.}

Lemma 2.9. Assume that $\# \mathbb{K}>2$. Let $\lambda \in \mathbb{K}$. Assume that $A:=\lambda I_{3}+E_{2,3}$ has trace zero. Let $B \in \mathfrak{s l}_{3}(\mathbb{K}) \backslash\{0\}$, and set $\mathcal{H}:=\{B\}^{\perp}$. Then, $A \in[\mathcal{H}, \mathcal{H}]$.

Proof. We assume that $A \notin[\mathcal{H}, \mathcal{H}]$ and search for a contradiction. By point (a) in Lemma 2.2 for every basis $\mathbf{B}=(x, y, z)$ of $\mathbb{K}^{3}$ for which $P_{\mathbf{B}}^{-1} A P_{\mathbf{B}}$ is upper-triangular, we find $B x \in \operatorname{span}(x, y)$. In particular, for every basis $(x, y)$ of $\operatorname{span}\left(e_{1}, e_{2}\right)$, the triple $\left(x, y, e_{3}\right)$ qualifies, whence $B x \in \operatorname{span}(x, y)=\operatorname{span}\left(e_{1}, e_{2}\right)$. It follows that $\operatorname{span}\left(e_{1}, e_{2}\right)$ is stable under $B$. As $z \mapsto A z$ is also represented by an upper-triangular matrix in the basis $\left(e_{2}, e_{3}, e_{1}\right)$, one finds $B e_{2} \in \operatorname{span}\left(e_{2}, e_{3}\right)$, whence $B e_{2} \in \mathbb{K} e_{2}$. Thus, $B$ has the following shape:

$$
B=\left[\begin{array}{lll}
a & 0 & d \\
b & c & e \\
0 & 0 & f
\end{array}\right]
$$

From there, we split the discussion into two main cases.

Case 1. $\lambda=0$.

Using $\left(e_{2}, e_{1}, e_{3}\right)$ as our new basis, we are reduced to the case when

$$
A=\left[\begin{array}{lll}
0 & 0 & 1 \\
0 & 0 & 0 \\
0 & 0 & 0
\end{array}\right] \text { and } B=\left[\begin{array}{lll}
? & ? & ? \\
0 & ? & ? \\
0 & 0 & ?
\end{array}\right]
$$

Then, one checks that $\left[J_{2}, E_{2,3}\right]=A$, and $\operatorname{tr}\left(J_{2} B\right)=0=\operatorname{tr}\left(E_{2,3} B\right)$. This yields 
$A \in[\mathcal{H}, \mathcal{H}]$, contradicting our assumptions.

Case 2. $\lambda \neq 0$.

As we can replace $A$ with $\lambda^{-1} A$, which is similar to $I_{3}+E_{2,3}$, no generality is lost in assuming that $\lambda=1$. According to principle (2) of Section 2.1 no further generality is lost in subtracting a scalar multiple of $A$ from $B$, to the effect that we may assume that $f=0$ and $B \neq 0$ (if $B$ is a scalar multiple of $A$, then the same principle combined with the Albert-Muckenhoupt theorem shows that $A \in[\mathcal{H}, \mathcal{H}])$. As $\operatorname{tr} B=0$, we find that

$$
B=\left[\begin{array}{ccc}
a & 0 & d \\
b & -a & e \\
0 & 0 & 0
\end{array}\right]
$$

Note finally that $\mathbb{K}$ must have characteristic 3 since $\operatorname{tr} A=0$.

Subcase 2.1. $b \neq 0$.

As the problem is unchanged in multiplying $B$ with a non-zero scalar, we can assume that $b=1$. Assume furthermore that $d \neq 0$. Let $(\alpha, \beta) \in \mathbb{K}^{2}$, and set

$$
C:=\left[\begin{array}{lll}
0 & 1 & 0 \\
\alpha & 0 & 1 \\
\beta & 0 & 0
\end{array}\right] \text {. }
$$

Note that $C$ is a cyclic matrix and

$$
C^{2}=\left[\begin{array}{lll}
\alpha & 0 & 1 \\
\beta & \alpha & 0 \\
0 & \beta & 0
\end{array}\right]
$$

Thus, $\operatorname{tr}(A C)=0, \operatorname{tr}(B C)=\beta d+1, \operatorname{tr}\left(A C^{2}\right)=2 \alpha+\beta=\beta-\alpha$ and $\operatorname{tr}\left(B C^{2}\right)=e \beta$. As $d \neq 0$, we can set $\beta:=-d^{-1}$ and $\alpha:=\beta$, so that $\beta \neq 0$ and $\operatorname{tr}(A)=\operatorname{tr}(A C)=$ $\operatorname{tr}\left(A C^{2}\right)=0$. Thus, $A \in \operatorname{im}\left(\operatorname{ad}_{C}\right)$ by Lemma 1.7, and on the other hand $C \in \mathcal{H}$. As $A \notin[\mathcal{H}, \mathcal{H}]$, it follows that $\mathcal{C}(C) \subset \mathcal{H}$, and hence, $\operatorname{tr}\left(B C^{2}\right)=0$. As $\beta \neq 0$, this yields $e=0$.

From there, we can find a non-zero scalar $t$ such that $d+t a \neq 0$ (because $\# \mathbb{K}>2$ ). In the basis $\left(e_{1}, e_{2}, e_{3}+t e_{1}\right)$, the respective matrices of $z \mapsto A z$ and $z \mapsto B z$ are $I_{3}+E_{2,3}$ and

$$
\left[\begin{array}{ccc}
a & 0 & d+t a \\
1 & -a & t \\
0 & 0 & 0
\end{array}\right]
$$

As $d+t a \neq 0$ and $t \neq 0$, we find a contradiction with the above line of reasoning. 
Therefore, $d=0$. Then, the matrices of $z \mapsto A z$ and $z \mapsto B z$ in the basis $\left(e_{1}, e_{2}, e_{3}+e_{1}\right)$ are, respectively, $I_{3}+E_{2,3}$ and $\left[\begin{array}{ccc}a & 0 & a \\ 1 & -a & e+1 \\ 0 & 0 & 0\end{array}\right]$. Applying the above proof in that new situation yields $a=0$. Therefore,

$$
B=\left[\begin{array}{lll}
0 & 0 & 0 \\
1 & 0 & e \\
0 & 0 & 0
\end{array}\right]
$$

With $\left(e_{3}-e e_{1}, e_{1}, e_{2}\right)$ as our new basis, we are finally left with the case when

$$
A=\left[\begin{array}{lll}
1 & 0 & 0 \\
0 & 1 & 0 \\
1 & 0 & 1
\end{array}\right] \text { and } B=\left[\begin{array}{lll}
0 & 0 & 0 \\
0 & 0 & 0 \\
0 & 1 & 0
\end{array}\right]
$$

Set

$$
C:=\left[\begin{array}{lll}
1 & 0 & 1 \\
1 & 1 & 0 \\
0 & 1 & 0
\end{array}\right]
$$

and note that $C$ is cyclic and

$$
C^{2}=\left[\begin{array}{ccc}
1 & 1 & 1 \\
-1 & 1 & 1 \\
1 & 1 & 0
\end{array}\right]
$$

One sees that $\operatorname{tr}(A)=\operatorname{tr}(A C)=\operatorname{tr}\left(A C^{2}\right)=0$, and hence, $A \in \operatorname{im}\left(\operatorname{ad}_{C}\right)$ by Lemma 1.7. On the other hand, $\operatorname{tr}(B C)=0$. As $A \notin[\mathcal{H}, \mathcal{H}]$, one should find $\operatorname{tr}\left(B C^{2}\right)=0$, which is obviously false. Thus, we have a final contradiction in that case.

Subcase 2.2. $b=0$.

Assume furthermore that $a \neq 0$. Then, in the basis $\left(e_{1}+e_{2}, e_{2}, e_{3}\right)$, the respective matrices of $z \mapsto A z$ and $z \mapsto B z$ are $I_{3}+E_{2,3}$ and $\left[\begin{array}{ccc}a & 0 & d \\ -2 a & -a & e-d \\ 0 & 0 & 0\end{array}\right]$. This sends us back to Subcase 2.1, which leads to another contradiction. Therefore, $a=0$.

If $d=0$, then we see that $B \in \operatorname{span}\left(I_{n}, A\right)$, and hence, principle (2) from Section 2.1 combined with Theorem 1.1 shows that $A \in[\mathcal{H}, \mathcal{H}]$, contradicting our assumptions. Thus, $d \neq 0$. Replacing the basis $\left(e_{1}, e_{2}, e_{3}\right)$ with $\left(d e_{1}+e e_{2}, e_{2}, e_{3}\right)$, we are reduced to the case when

$$
A=\left[\begin{array}{lll}
1 & 0 & 0 \\
0 & 1 & 1 \\
0 & 0 & 1
\end{array}\right] \text { and } B=\left[\begin{array}{lll}
0 & 0 & 1 \\
0 & 0 & 0 \\
0 & 0 & 0
\end{array}\right]
$$


In that case, we set

$$
C:=\left[\begin{array}{ccc}
0 & 0 & 0 \\
1 & 0 & 0 \\
0 & 1 & -1
\end{array}\right]
$$

which is a cyclic matrix with

$$
C^{2}=\left[\begin{array}{ccc}
0 & 0 & 0 \\
0 & 0 & 0 \\
1 & -1 & 1
\end{array}\right]
$$

so that $\operatorname{tr}(A)=\operatorname{tr}(A C)=\operatorname{tr}\left(A C^{2}\right)=0$ and $\operatorname{tr}(B C)=0$. As $\operatorname{tr}\left(B C^{2}\right) \neq 0$, this contradicts again the assumption that $A \notin[\mathcal{H}, \mathcal{H}]$. This final contradiction shows that the initial assumption $A \notin[\mathcal{H}, \mathcal{H}]$ was wrong.

2.6. Conclusion. Let $A \in \mathrm{M}_{n}(\mathbb{K})$ and $B \in \mathrm{M}_{n}(\mathbb{K}) \backslash\{0\}$, where $n \geq 3$ and $\# \mathbb{K} \geq 4$. Set $\mathcal{H}:=\{B\}^{\perp}$ and assume that $\operatorname{tr}(A)=0$ and $\operatorname{tr}(B)=0$. If $A$ is similar to $\lambda I_{3}+E_{2,3}$, then we know from Lemma 2.9 and principle (3) of Section 2.1 that $A \in[\mathcal{H}, \mathcal{H}]$. Otherwise, if $\left(I_{n}, A, B\right)$ is LLD then we know from Lemma 2.8 that $A \in[\mathcal{H}, \mathcal{H}]$. Using Lemma 2.3 , we conclude that $A \in[\mathcal{H}, \mathcal{H}]$ in every possible situation. This completes the proof of Theorem 1.4

\section{REFERENCES}

[1] A.A. Albert and B. Muckenhoupt. On matrices of trace zero. Michigan Math. J., 4:1-3, 1957.

[2] M. Brešar and P. Šemrl. On locally linearly dependent operators and derivations. Trans. Amer. Math. Soc., 351:1257-1275, 1999.

[3] M. Chebotar and P. Šemrl. Minimal locally linearly dependent spaces of operators. Linear Algebra Appl., 429:887-900, 2008.

[4] P.A. Fillmore. On similarity and the diagonal of a matrix. Amer. Math. Monthly, 76:167-169, 1969.

[5] T. Laffey and R. Reams. Integral similarity and commutators of integral matrices. Linear Algebra Appl., 197/198:671-689, 1994.

[6] C. de Seguins Pazzis. Local linear dependence seen through duality I. Preprint, arXiv: http://arxiv.org/abs/1306.1845, 2013.

[7] C. de Seguins Pazzis. Local linear dependence seen through duality II. Preprint, arXiv: http://arxiv.org/abs/1306.5722, 2013.

[8] C. de Seguins Pazzis. To what extent is a large space of matrices not closed under product? Linear Algebra Appl., 435:2708-2721, 2011.

[9] K. Shoda. Einige Sätze über Matrizen. Jap. J. Math., 13:361-365, 1936.

[10] A. Stasinski. Similarity and commutators of matrices over principal ideal rings. Preprint, arXiv: http://arxiv.org/abs/1211.6872, 2012.

[11] R.C. Thompson. Matrices with zero trace. Israel J. Math., 4:33-42, 1966. 